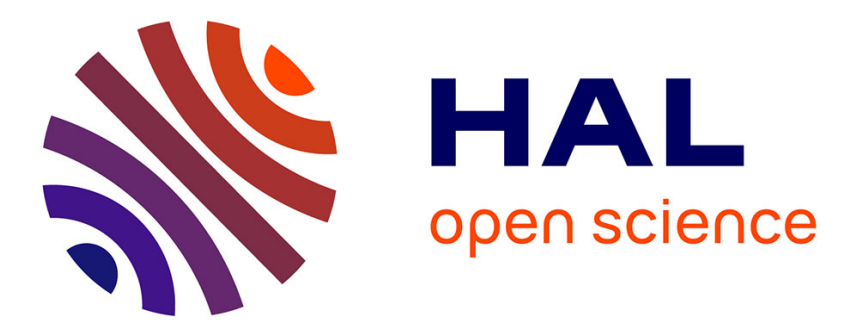

\title{
A simple observer based controller for the stabilization of systems with two unstable poles plus time delay
}

David Novella Rodriguez, Basilio del Muro-Cuellar, Olivier Sename

\section{To cite this version:}

David Novella Rodriguez, Basilio del Muro-Cuellar, Olivier Sename. A simple observer based controller for the stabilization of systems with two unstable poles plus time delay. MED 2011 - 19th

Mediterranean Conference on Control and Automation, Jun 2011, Corfou, Greece. pp.1522-1527, 10.1109/MED.2011.5983130 . hal-00682128

\section{HAL Id: hal-00682128 \\ https://hal.science/hal-00682128}

Submitted on 23 Mar 2012

HAL is a multi-disciplinary open access archive for the deposit and dissemination of scientific research documents, whether they are published or not. The documents may come from teaching and research institutions in France or abroad, or from public or private research centers.
L'archive ouverte pluridisciplinaire HAL, est destinée au dépôt et à la diffusion de documents scientifiques de niveau recherche, publiés ou non, émanant des établissements d'enseignement et de recherche français ou étrangers, des laboratoires publics ou privés. 


\title{
A Simple Observer Based Controller for the Stabilization of Systems with Two Unstable Poles Plus Time Delay.*
}

\author{
David Novella Rodríguez $^{\dagger}$, Basilio Del Muro Cuéllar ${ }^{\dagger}$ and Olivier Sename f $^{\ddagger}$ \\ † Escuela Superior de Ingeniería Mecánica y Eléctrica, Unidad Culhuacan, \\ Instituto Politécnico Nacional, Santa Ana 1000, México D.F., 04430, México. \\ (e-mail: dnovellar@gmail.com,bdelmuro@yahoo.com). \\ $\ddagger$ GIPSA-Lab Department of Control Systems, Grenoble INP, ENSE3, \\ BP46 38402, Saint Martin d'Hères Cedex, GRENOBLE, FRANCE. \\ (e-mail: olivier.sename@gipsa-lab.grenoble-inp.fr).
}

\begin{abstract}
This work considers the problem of the stabilization of linear time invariant systems with two unstable poles plus time delay. For this, we will proposed a simple observer based controller in order to stabilize the system. A robustness analysis with respect to the delay uncertainty is presented as well as numerical examples and electronic implementation of the proposed schema in order to illustrate the performance of the closed loop system.
\end{abstract}

\section{INTRODUCTION.}

Time-delay is the property of a physical system by which the response to an applied signal is delayed in its effect. Systems with delays are very common in the world, they appears in various systems as biological, ecological, social, engineering systems, etc., and are due to several mechanisms like material or energy transport, recycling loops, etc,. In addition, actuators, sensors and field networks that are involved in feedback loops usually introduce such delays, [16]. Also delays can be used in modeling reduction where highorder (finite-dimensional) systems are approximated (in some norm sense) by low order systems with delays, [13]. It is known that time-delay is often a source of complex behaviors (oscillations, instability, bad performance), in many dynamic systems, and thus considerable attention has been paid on the stability analysis and controller design of time delay systems. Hence, there exist a great motivation to study of delay effects on dynamical systems properties for two main reasons: first to understand how the delay presence may deteriorate the behavior of the system, and second to control their effects for better performance achieved on closed-loop systems, [5], [1].

Several control strategies have been developed to deal with delayed systems. A common approach is to approximate the time-delay operator by means of a Taylor or Pade series which could leads to a non minimum-phase system with rational transfer function representation. Another classical control approaches for time-delay systems include ProportionalIntegral-Derivative (PID) control, classical smith predictors (SPC), etc, [8], [14].

Open-loop unstable processes arise frequently in the chemical and biological systems and are fundamentally difficult to

\footnotetext{
*This work was supported in part by CONACyT-México, Under Grant 61713 .
}

control. Unstable time-delay systems represent a challenge for control design, for instance the SPC scheme has not a stabilization step, which restricts its application to open-loop stable plants. To get over this problem, some modifications of the SPC original structure have been proposed to deal with non-stable delayed process, for instance, [11] has presented an efficient modification to the Smith predictor in order to control unstable first order system plus time delay. With a different perspective, [7] proposes a modification to the original Smith Structure in order to deal with unstable first order delayed systems. Using a similar structure, the result is extended to delayed high order systems [6]. In the last works, a robustness analysis is done concluding that for unstable dead time dominant systems, the closed-loop system can be unstabilized with an infinitesimal value of the modeling error, i.e., that robustness is strongly dependent on the relationship $\tau / \tau_{u n}$, where $\tau$ is the process time delay and $\tau_{u n}$ the dominant unstable time-constant. For the control scheme proposed in both latter works, it can be easily proven that in the case of unstable plants, the internal stability is not guaranteed. In fact it is obtained an unstable estimation error and, as a result, a minimal initial condition difference between the original plant and the model produces an internal unbounded signal. Notice that in a practical situation it is not possible to exactly measure the initial condition of the plant to assign the same value to the model considered on the modified Smith compensator.

Many chemical and biological systems exist whose dynamics present second-order behavior. Continuous stirred tank reactors, polymerization reactors and bioreactors are inherently unstable by design, these types of systems can be modeled as open-loop unstable second-order plus time delay models, [12], [9]. This paper is concerned with the stabilization problem of systems with two unstable poles plus time delay. The control schema relies on an observer-based structure with a memory observer and a memoryless state feedback. On the contrary of modified Smith predictors, the scheme only contains discrete time delay (and not distributed ones) which makes easy its practical implementation (see [16] for details on numerical implementation of modified Smith predictor scheme). This paper is organized as follows, the Section 2 is dedicated to the problem formulation. The 
Section 3 yields the preliminaries results used to obtain the main result of this work. An observer based controller is proposed in order to stabilize the unstable system in the Section 4, also the stability conditions of the proposed controller schema are stated. Then, in section 5 a robustness analysis for the observer based schema with respect to the uncertainty in the delay operator is presented. Numerical simulations are presented in order to show the controller performance and the control strategy is implemented on a real electronic plant built with operational amplifiers. Finally conclusions and future perspectives are stated.

\section{Problem Statement.}

Consider the following class of single-input single-output (SISO) linear systems with delay at the input:

$$
\frac{Y(s)}{U(s)}=\frac{N(s)}{D(s)} e^{-\tau s}=G(s) e^{-\tau s} .
$$

Where $U(s)$ and $Y(s)$ are the input and output signals respectively, $\tau \geq 0$ is the time delay, $N(s)$ and $D(s)$ are polynomials in the complex variable $s$ and $G(s)$ is the delayfree transfer function. Notice that with respect to the class of systems (1) a traditional control strategy based on an output feedback of the form:

$$
U(s)=[R(s)-Y(s)] Q(s) .
$$

Produces a closed-loop system given by:

$$
\frac{Y(s)}{R(s)}=\frac{Q(s) G(s) e^{-\tau s}}{1+Q(s) G(s) e^{-\tau s}}
$$

Where the exponential term $e^{-\tau s}$ located at the denominator of the transfer function (3) leads to a system with an infinite number of poles and where the closed-loop stability properties must be carefully stated. This work proposes an observer based control scheme in order to stabilize a system characterized by the following transfer function:

$$
\frac{Y(s)}{U(s)}=\frac{\alpha}{(s-a)(s-b)} e^{-\tau s} .
$$

Where, without loss of generality, $a \geq b>0, \tau>0$. The control schema proposed has been designed based on a traditional observer theory, hence, only the plant model and two static gains are enough to get an adequate estimation of an internal delay free variable which will be used in the final stabilizing control schema.

\section{Preliminary Results}

Preliminary results are presented, which will be used later in order to state the stability conditions of the studied system.

Consider the following unstable first order system plus time delay:

$$
\frac{Y(s)}{U(s)}=G(s) e^{-\tau s}=\frac{\alpha}{s-\sigma} e^{-\tau s}, .
$$

With $\sigma>0$, and a proportional output feedback control as follows:

$$
U(s)=R(s)-k Y(s) .
$$

Which produces a closed-loop system:

$$
\frac{Y(s)}{R(s)}=\frac{\alpha e^{-\tau s}}{s-\sigma+k \alpha e^{-\tau s}} .
$$

The following result has been widely studied in the literature and the proof can be easily obtained by considering different approaches as a classical frequency domain. An alternative simple proof based on a discrete time approach is shown in [3].

Lemma 1: Consider the delayed system (5) and the proportional output feedback (6). Then, there exists a proportional gain $k$ such that the closed loop system (7), is stable if and only if $\tau<\frac{1}{\sigma}$.

Now, consider the system characterized by:

$$
\frac{Y(s)}{U(s)}=G(s) e^{-\tau s}=\frac{\alpha}{(s-\sigma)(s+\phi)} e^{-\tau s} .
$$

With $\sigma, \phi>0$. Note that the system has only one unstable pole. With the proportional output feedback (6), we get a closed-loop system as follows:

$$
\frac{Y(s)}{R(s)}=\frac{\alpha e^{-\tau s}}{[(s-\sigma)(s+\phi)]+k \alpha e^{-\tau s}} .
$$

Lemma 2: Consider the delayed system (8) and the proportional output feedback (6). Then, there exists a proportional gain $k$ such that the closed loop system (9), is stable if and only if $\tau<\frac{1}{\sigma}-\frac{1}{\phi}$.

The proof of this result can easily be obtained by mean of different approaches, as the frequency domain based approach shown in [4].

\section{Control Strategy Proposed.}

Consider the class of systems studied in this work and characterized by the transfer function (4) with $a, b>0$ and assuming without lost of generality $a \geq b$. An observer based control strategy is proposed, which allows to get an estimation of the internal variables of the system to be used as control signals for the real process.

As a first step, the stability conditions for the controller and the observer systems are stated separately. This conditions will be used later to submit the observer based controller closed loop stability conditions.

\section{A. Controller Schema.}

Now, taking into account the proportional state feedback control strategy shown in the Figure 1.

Lemma 3: Consider the delayed system (4), and the state feedback controller shown in Figure 1. There exist constants $k_{1}$ and $k_{2}$ such that the closed-loop system is stable if and only if $\tau<\frac{1}{b}$.

Proof:

Sufficiency. Let us consider $\tau<\frac{1}{b}$. Then, $\tau=\frac{1}{b}-\beta$, for some $\beta>0$. Therefore, there exists $k_{1}$ such that $\beta>$ $\frac{1}{\alpha k_{1}-a}>0$. Then $\tau<\frac{1}{b}-\frac{1}{\alpha k_{1}-a}$. From Lemma 2, where 
$\sigma=b$ and $\phi=\alpha k_{1}-a$ there exists $k_{2}$ such that the closed loop system shown in Figure 1 is stable.

Necessity. Consider the delayed system (4), and the state feedback controller shown in Figure 1, with constants $k_{1}$ and $k_{2}$ such that the closed-loop system is stable. The closed loop transfer function can be written as follows:

$$
\frac{Y(s)}{R(s)}=\frac{\alpha e^{-\tau s}}{[(s-b)(s+\phi)]+k_{2} \alpha e^{-\tau s}},
$$

with $\phi=\alpha k_{1}-a$. It is well known that a $k_{2}$ that stabilizes the delayed system (10) must also stabilize the delay free system (see for instance [2] or [5]), which implies that $\phi$ $>0$. Indeed, from Lemma 2, $\tau<\frac{1}{\sigma}-\frac{1}{\phi}$ with $\sigma=b$ (note that $\phi>0$ is a free parameter function of $k_{1}$ ). Let us consider $\beta>\frac{1}{\phi}>0$, denoting $\beta=\frac{1}{\sigma}-\tau$, therefore $\tau=\frac{1}{\sigma}-\beta<$ $\frac{1}{\sigma}=\frac{1}{b}$.

Note that a root locus and frequency domain analysis can be used to compute proper constant gains $k_{1}$ and $k_{2}$ in order to stabilize the proportional states feedback scheme.

\section{B. Observer Scheme.}

In most of the practical applications, some of the state variables may not be measured. Thus, an observer, based on an output injection strategy is proposed, it can be represented as in Figure 2. The stability of the observer can be tackled as follows.

Lemma 4: Consider the delayed system (4), and the static output injection scheme shown in Figure 2. There exist constants $g_{1}$ and $g_{2}$ such that the closed-loop system is stable if and only if

Proof:

$$
\tau<\frac{1}{a} .
$$

The proof can be easily derived from a dual procedure of the previous result.

As in the controller design, the computation of the proportional gains $g_{1}$ and $g_{2}$ can be solved by mean of a root locus and frequency domain analysis.

\section{Observer-Based Controller.}

Finally, the main result of this work is presented, we propose an observed based controller as in the Figure 3, where the observer allows to estimate the state variables, to be use in state feedback controller. The authors would like

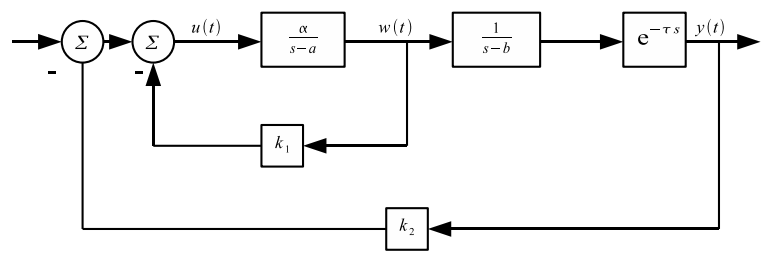

Fig. 1. State Feedback Scheme.

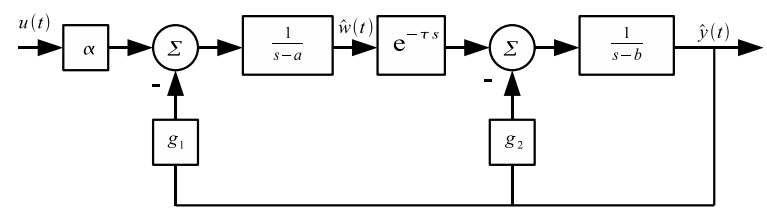

Fig. 2. Output-Injection Scheme of the Observer.

to stress that, in the proposed scheme, only four proportional gains are sufficient to get a stable closed loop behavior. As a consequence of the previous results, the following lemma can be stated.

Lemma 5: Consider the observer based controller scheme shown in Figure 3. There exist $k_{1}, k_{2}, g_{1}$ and $g_{2}$ such that the closed-loop system is stable if and only if $\tau<\frac{1}{a}$.

Proof: As a first step, in order to ensure an accurate estimation of the states variables, let us demonstrate that the error signal converges asymptotically to zero, i.e., $\lim _{t \rightarrow \infty}[\hat{w}(t)-w(t)]=0$ if and only if $\tau<\frac{1}{a}$. Consider the state space representation of the system (4) characterized by the following equation:

$$
\begin{gathered}
\dot{x}(t)=A_{0} x(t)+A_{1} x(t-\tau)+B u(t) \\
y(t)=C x(t)
\end{gathered}
$$

. With $x(t)=\left[\begin{array}{ll}w(t) & y(t)\end{array}\right]^{T}$. Where,

$$
\begin{array}{cc}
A_{0}=\left[\begin{array}{ll}
a & 0 \\
0 & b
\end{array}\right] \quad, \quad A_{1}=\left[\begin{array}{ll}
0 & 0 \\
1 & 0
\end{array}\right] \\
B=\left[\begin{array}{l}
\alpha \\
0
\end{array}\right] \quad, \quad C=\left[\begin{array}{ll}
0 & 1
\end{array}\right] .
\end{array}
$$

Note that the state space representation characterized by (11) can be returned to its transfer function representation by mean of:

$$
\frac{Y(s)}{U(s)}=C\left(s I-\left(A_{0}+A_{1} e^{-\tau s}\right)\right)^{-1} B .
$$

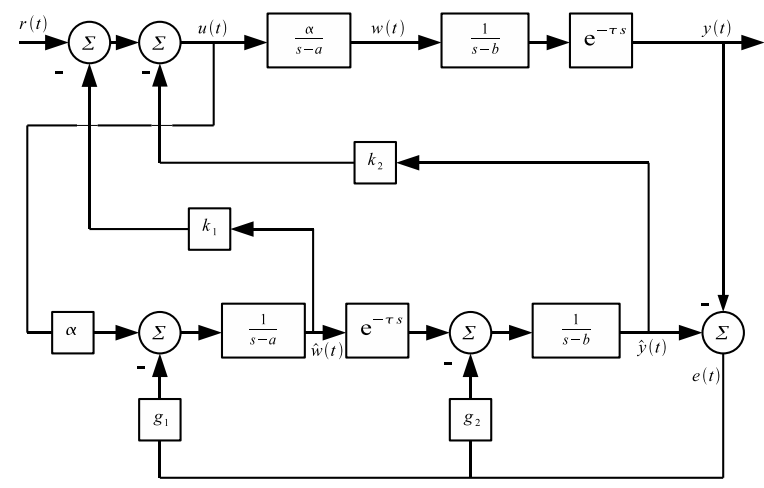

Fig. 3. Control Strategy Proposed. 
Which brings us back to the delayed transfer function (4). The dynamics of the estimated states and the control law can be described as follows.

$$
\begin{gathered}
\dot{\hat{x}}(t)=A_{0} \hat{x}(t)+A_{1} \hat{x}(t-\tau)+B u(t)-G(C \hat{x}(t)-y(t)) \\
u(t)=K \hat{x}(t) .
\end{gathered}
$$

Where $\hat{x}(t)$ is the estimated state of $x(t)$, and the proportional gains are defined by $K=\left[\begin{array}{ll}k_{1} & k_{2}\end{array}\right]$ and $G=\left[\begin{array}{ll}g_{1} & g_{2}\end{array}\right]^{T}$.

Let be $e(t):=x(t)-\hat{x}(t)$, then we have:

$$
\dot{e}(t)=\dot{x}(t)-\dot{\hat{x}}(t)=\left(A_{0}-G C\right) e(t)+A_{1} e(t-\tau) .
$$

And the controlled system:

$$
\dot{x}(t)=A_{0} x(t)+A_{1} x(t-\tau)+B K \hat{x}(t) .
$$

Noting $x_{e}=[x(t) e(t)]^{T}$ and after a simple manipulation of variables we have the following closed loop system with the observer and the controller proposed in the Figure 3:

$$
\begin{gathered}
\dot{x_{e}}(t)=\left[\begin{array}{cc}
A_{0}+B K & -B K \\
0 & A_{0}-G C
\end{array}\right] x_{e}(t)+\cdots \\
\cdots+\left[\begin{array}{cc}
A_{1} & 0 \\
0 & A_{1}
\end{array}\right] x_{e}(t-\tau) \\
y(t)=\left[\begin{array}{cc}
C & 0
\end{array}\right] x_{e}(t) .
\end{gathered}
$$

It is easy to see that the observer and the controller can be designed separately, i.e. satisfies the separation principle. Hence, the stability of the observer scheme is sufficient to assure the error convergence, i.e. there exist proportional gains $g_{1}$ and $g_{2}$ such that $\lim _{t \rightarrow \infty}[\hat{w}(t)-w(t)]=0$ if and only if $\tau<\frac{1}{a}$.

Then, considering the fact of the observer and controller can be designed separately and reminding the stability conditions stated previously in Lemmas 4 and 3, is clear that the observer stability condition is more restrictive than the controller stability condition, i.e. $\frac{1}{a}<\frac{1}{b}$. Therefore, there exist $k_{1}, k_{2}, g_{1}$ and $g_{2}$ such that the closed-loop system is stable if and only if $\tau<\frac{1}{a}$.

\section{Examples.}

The following numerical examples illustrate the performance obtained by main of the observed based controller proposed.

Example 1. Consider the unstable delayed system characterized for the following transfer function:

$$
\frac{Y(s)}{U(s)}=\frac{1}{(s-0.7)(s-0.4)} e^{-s} .
$$

Let be $a=0.7, b=0.4, \alpha=1$ and $\tau=1$, it is clear that the stability conditions given in Lemma 5 are satisfied, therefore there exist an observer based structure with proportional gains $k_{1}, k_{2}, g_{1}$ and $g_{2}$ such that the resulting closed-loop system is stable due to $\tau=1<$ $\frac{1}{a}=1.428$. As was demonstrated before, the control scheme 3 holds the separation property, hence the design of the controller and the observer is independent. First, from the proof of Lemma 3, the gain $k_{1}$ must be larger than $a / \alpha$. On the other hand, to ensure the existence of a proportional gain $k_{2}$ such that the closed loop system is stable, from Lemma 2

$$
k_{1}>\frac{1}{\alpha\left(\frac{1}{b}-\tau\right)}+\frac{a}{\alpha} .
$$

As the value $k_{1}$ can be as large as we wish, the gain chosen is $k_{1}=100.7$, form this, after a frequency domain analysis, Nyquist stability criterion for instance, we can compute the gain $k_{2}$ such that the controller scheme shown in the Figure 3 is stable, for this example, $40<k_{2}<131.06$.

The procedure for compute the observer gains $g_{1}$ and $g_{2}$ is quite similar, the proportional gain $g_{2}$ must be larger than $b$ to ensure stability of the system. From Lemma 2 we know that

$$
g_{2}>\frac{1}{\left(\frac{1}{a}-\tau\right)}+b .
$$

As the value $g_{2}$ can be as large as we wish, the gain chosen is $g_{2}=100.4$, form this, after a frequency domain analysis, we can compute the gain $g_{1}$, such that the observer scheme shown in the Figure 2 is stable, for this example, $69.93<g_{1}<113.89$.

Hence, the constant gains computed for this example are $k_{1}=100.7, \quad k_{2}=90, g_{1}=80$ and $g_{2}=100.4$. The Figure 4 illustrate the performance of the observer based controller in numerical simulations, the output and the error are shown respectively. The continuous line indicates the output of the closed loop system with identical initial conditions between $x(t)$ and $\hat{x}(t)$. The dashed line point to the system performance whit different initial conditions $(w(0)-\hat{w}(0)=0.5)$.

Below, for the same example, the observed based controller is implemented by mean of the Data Acquisition system Sensoray 626, using its analog inputs/ouputs as sensors
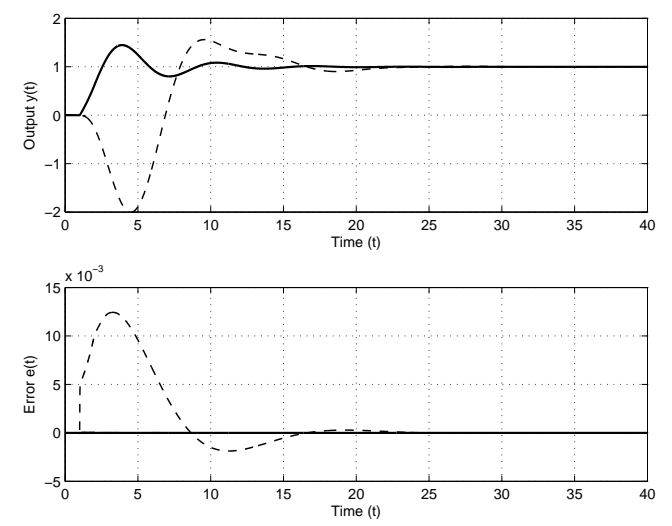

Fig. 4. Numerical Simulation Results. 


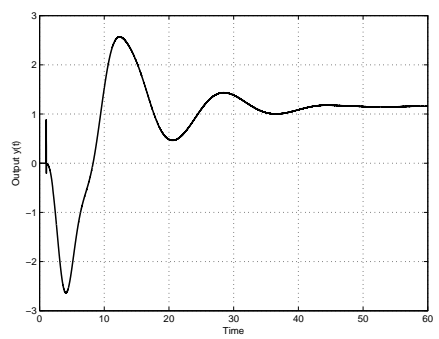

Fig. 5. Practical Implementation.

and actuators communicated with a computer through the MATLAB Real Time Workshop Toolbox. The real electronic plant is built with commercial operational amplifiers, resistors, and capacitors. The stable output $y(t)$ of the closed loop system is shown in the Figure 5.

Remark 1: Notice that the controller have a efficient performance regardless the different initial conditions between the original process and the observer, and the parametrical variations due to the use of commercial electronic devices.

\section{Robustness Analysis With Respect To Delay UNCERTAINTY.}

No mathematical representation can exactly model a physical system. For this reason we must be aware of how modeling errors might adversely affect the performance of a control system. Here, we refer to robustness with respect to delay to the fact that the exact value of the delay is not known a priori.

Therefore, we assume now that the delay is uncertain, $i$. $e$. the delay of the real system is $\tau=d(1+\delta \theta)$ and may be different from the one used in the observer (the nominal delay $d$ ). Consider the delay uncertainty in the original process, and the design of the observer taking into account the nominal time delay, i.e.,

$\dot{x}(t)=A_{0} x(t)+A_{1} x(t-\tau)+B u(t)$

$\dot{\hat{x}}(t)=A \hat{x}(t)+A_{1} \hat{x}(t-d)+B u(t)-G(C \hat{x}(t)-y(t))$

$u(t)=K \hat{x}(t)$.

Let us consider the observer-controller system with the form (18) and its extended closed loop system considering the delay uncertainty characterized as follows:

$$
\begin{gathered}
\dot{x}_{e}(t)=\left[\begin{array}{cc}
A_{0}+B K & -B K \\
0 & A_{0}-G C
\end{array}\right] x_{e}(t)+ \\
{\left[\begin{array}{cc}
A_{1} & 0 \\
A_{1} & 0
\end{array}\right]\left(x_{e}\right)_{\tau}(t-\tau)+\left[\begin{array}{cc}
0 & 0 \\
-A_{1} & A_{1}
\end{array}\right]\left(x_{e}\right)_{d}(t-d),} \\
=\mathcal{A}_{0} x_{e}(t)+\mathcal{A}_{\tau}\left(x_{e}\right)_{\tau}(t-\tau)+\mathcal{A}_{d}\left(x_{e}\right)_{d}(t-d) .
\end{gathered}
$$

Note that when the delay value of the actual process matches to the nominal one, the extended closed loop system
(20) is equivalent to the nominal closed loop system (16), [15]. Now modeling the time delay with a multiplicative uncertainty as $\tau=d(1+\delta \theta)$, with $\theta$ normalized, i.e. $|\theta| \leq 1$ we obtain.

$$
e^{-s \tau}=e^{-s d(1+\delta \theta)}=e^{-s d} e^{-s d \delta \theta}=e^{-s d}(1-\Delta) .
$$

With $\Delta(s)=1-e^{-s d \delta \theta}$. Therefore the characteristic polynomial of the above system is given by:

$$
\Psi(s)=\operatorname{det}\left[\Psi_{0}(s)\right] \operatorname{det}\left[I_{n}+\Psi_{0}^{-1}(s) \mathcal{A}_{\tau} e^{-s d} \Delta(s)\right] .
$$

Where $\Psi_{0}(s)=I_{2 n}-\mathcal{A}_{0}-\left(\mathcal{A}_{\tau}+\mathcal{A}_{d}\right) e^{-s d}$. Now, when the design of the controller and the observer ensures that the nominal extended system is stable, $i$. e. $\operatorname{det}\left[\Psi_{0}(s)\right]$ is stable, the perturbed closed loop system remains stable if $\operatorname{det}\left[I_{n}+\Psi_{0}^{-1}(s) \mathcal{A}_{\tau} e^{-s d} \Delta(s)\right]$ does not change sign when $s$ sweeps the imaginary axis. Invoking Rouche's Theorem, it follows that the condition for stability is:

$$
\left\|Q_{d}(s) \Delta(s)\right\|_{\infty}<1
$$

Where $Q_{d}(s)=\Psi_{0}^{-1}(s) \mathcal{A}_{\tau} e^{-s d}$. Note that the term $1-$ $e^{-s d \delta \theta}=\left.s d \delta \theta e^{-s d \delta \theta / 2}\right|_{s=j \omega} \operatorname{sinc} \frac{\omega d \delta \theta}{2 \pi}$. Hence, on the imaginary axis, $\left|1-e^{-s d \delta \theta}\right| \leq|s d \delta \theta|$, then if one can show stability for $1-e^{s d \delta \theta}$ replacing it by $s d \delta \theta$ in the analysis, then stability for the uncertain system will follow. Note that this amounts to replacing the delayed term by its first order Taylor expansion. This means that the maximal uncertainty bound $\delta$ that preserves stability for the worst case, i.e. $\theta=1$, is determined by:

$$
\delta_{\max }=\frac{1}{\left\|s d e^{-s d} \Psi_{0}^{-1}(s) \mathcal{A}_{\tau}\right\|_{\infty}} .
$$

Then for all $|\theta| \leq 1$ and the uncertainty bound $\delta_{\max }$ the determinant has a fixed sign, implying the absence of zero crossings, and henceforth the stability of the perturbed system (provided the nominal one is stable) [10], [15].

Example 2. In order to illustrate the above robustness analysis we can consider the example given in the section IV, and characterized by the following transfer function:

$$
\frac{Y(s)}{U(s)}=\frac{1}{(s-0.7)(s-0.4)} e^{-s} .
$$

Let us choose $a=0.7, b=0.4, \alpha=1$ and $\tau=1$, the constant gains, already computed, to stabilize the system are $k_{1}=100.7, k_{2}=90, g_{1}=80$ and $g_{2}=100.4$. Therefore, with (24) we can compute $\delta_{\max }=0.057023$, the maximum uncertainty bound of the delay.

The Figure 6 shown the performance of the closed loop system when there is not uncertainty in the delay value (continuos line), and the behavior when the maximum uncertainty bound is present in the system (dash line). The following table shows the relation between the the size of the time delay $d$ of the nominal system and the maximal uncertainty bound $\delta_{\max }$ in the above example. The terms $\tau_{\min }$ and $\tau_{\max }$ indicate the minimal and maximal value respectively of the 


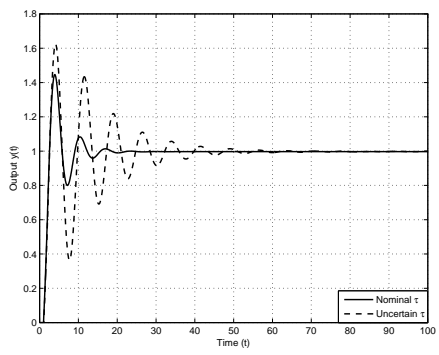

Fig. 6. Robustness Analysis W.R.T. Delay Uncertainty

delay term $\tau$, such that the closed loop system with nominal delay $d$, remains stable.

\begin{tabular}{|c|c|c|c|}
\hline$d$ & $\delta_{\max }$ & $\tau_{\min }$ & $\tau_{\max }$ \\
\hline 0.1 & 3.855741 & - & 0.485574 \\
\hline 0.2 & 1.739842 & - & 0.547968 \\
\hline 0.3 & 1.026864 & - & 0.608059 \\
\hline 0.4 & 0.665379 & 0.133848 & 0.666152 \\
\hline 0.5 & 0.441873 & 0.279064 & 0.720936 \\
\hline 0.6 & 0.293497 & 0.423902 & 0.776098 \\
\hline 0.7 & 0.194797 & 0.563642 & 0.836358 \\
\hline 0.8 & 0.129188 & 0.696650 & 0.903350 \\
\hline 0.9 & 0.085734 & 0.822839 & 0.977161 \\
\hline 1.0 & 0.057023 & 0.942977 & 1.057023 \\
\hline
\end{tabular}

TABLE I

ROBUST ANALYSIS WITH DIFFERENT TIME DELAY SIZE.

Remark 2: Note that the stabilizing gains computed for the observer based controller for the nominal time delay $d$ are able to preserve stability of the closed loop system with a nominal delay belongs to the interval $\left[\begin{array}{ll}0 & d\end{array}\right]$, the analysis of the stability sets and regions are dealt in [5] and [2]

\section{CONCLUSIONS.}

An observer based controller is proposed in order to stabilize a linear-time invariant system with two unstable poles plus time delay. The conditions that ensure existence of the control scheme and the stability of the closed loop system are stated. The scheme is simple and may be easily implemented. Numerical examples illustrate the performance of the controller under ideal conditions, an implementation of the observer based controller is presented by mean of an electronic plant built with operational amplifiers and the Data Acquisition system Sensoray 626. This example shows the controller performance working under different initial conditions between the plant and the observer, as well as the parametrical variations introduced by the use of commercial devices, also an robustness analysis is presented to show the effects of the modeling errors of the delay operator. The extension of the presented results to design an observer based controller to high order systems with two unstable poles and its robustness analysis are taken into account as a future work.

\section{REFERENCES}

[1] Keqin Gu, Vladimir L. Kharitonov, and Jie Chen, Stability of TimeDelay Systems, Birk"häuser, 2003.
[2] Efim Malakhovski and Leonid Mirkin, On Stability of Second-order Quasi-polynomials with a Single Delay, Automatica 42 (2006), 10411047.

[3] B. Del Muro, J.F. Márquez, M. Velasco, and J. Alvarez, Stabization strategy for unstable first order linear systems with large time delay, International Conference on Modelling Identification and Control, ICMIC (2008)

[4] B. Del Muro-Cuéllar, O. González-Nagera, and Y. A. Pedraza, Stabilization of high-order systems with delay using a predictor schema, Circuits and Systems, Midwest Symposium on 0 (2009), 337-340.

[5] Silviu-Iulian Niculescu, Delay Effects on Stability: A Robust Control Approach, Springer, 2001.

[6] J. E. Normey-Rico and E. F. Camacho, Unified approach for robust dead-time compesator design, Journal of Process Control 19 (2009), 38-47.

[7] Julio E. Normey-Rico and Eduardo. F. Camacho, Dead-time compensators: A survey, Control Engineering Practice 16 (2008), no. 4, 407428.

[8] Z. J. Palmor, Time delay compensation smith predictor and its modifications, The control Handbook (1996), 224- 237.

[9] A. Seshagiri Rao and M. Chidambaram, Enhanced Two-Degrees-ofFreedom Control Strategy for Second-Order Unstable Processes with Time Delay, Ind. Eng. Chem. Res. 45 (2006), 3604-3614.

[10] Olivier Sename, Is a Mixed Design of Observer Controllers for Time Delay Systems Interesting?, Asian Journal of Control 9 (2007), no. 2 , 180-189.

[11] R. A. Seshagiri, V. S. R. Rao, and M. Chidambaram, Simple analytical design of modified smith predictor with improved performance for unstable first-order plus time delay (fodtp) processes, Ind. Eng. Chem. Res 46(13) (2007), 4561-4571.

[12] M. Shamsuzzoha, Jongpal Jeon, and Moonyong Lee, Improved Analytical PID Controller Design for the Second Order Unstable Process with Time Delay, 17th European Symposium on Computer Aided Process Engineering (2007).

[13] S. Skogestad, Simple Analytic Rules for Model Reduction and PID Controller Tunning, Journal of Process Control 13 (2003), 4.

[14] O. J. M. Smith, Close Control of Loops with Dead Time, Chem. Eng. Prog. 53 (1957), 217-219.

[15] Erik I. Verriest, Olivier Sename, and Pierdominico Pepe, Robust Observer-Controller for Delay Differential Systems, Procedings of the 41st IEEE Conference on Decision and Control (2002), 981-986.

[16] Qing-Chang Zhong, Robust Control of Time-Delay Systems, Springer, 2006 